\title{
HIV Type 1 Mother-to-Child Transmission Facilitated by Distinctive Glycosylation Sites in the gp120 Envelope Glycoprotein
}

\author{
Elly Baan, Anthony de Ronde, Stanley Luchters, ${ }^{2, \star}$ Joseph Vyankandondera, ${ }^{3}$ \\ Joep M. Lange, ${ }^{2}$ Georgios Pollakis, and William A. Paxton ${ }^{1}$
}

\begin{abstract}
The human immunodeficiency virus type 1 (HIV-1) characteristics associated with mother-to-child transmission (MTCT) are still poorly understood. We studied a cohort of 30 mothers from Rwanda infected with HIV-1 subtype $A$ or $C$ viruses of whom seven infected their children either during gestation or soon after birth. CD4 counts and viral load did not significantly differ between nontransmitting mother (NTM) versus transmitting mother (TM) groups. In contrast to earlier studies we not only analyzed and compared the genotypic characteristics of the V1-V5 region of the gp120 envelope of viruses found in TM and their infected children, but also included data from the NTM. No differences were found with respect to length and number of potential Nglycosylation sites (PNGS) in the V1-V2 and the V1-V5 region. We identified that viruses with a PNGS on positions AA234 and AA339 were preferably transmitted and that viruses with PNGS-N295 showed a disadvantage in transmission. We also showed that the frequency of PNGS-N339 in the viruses of TM and infected children was significantly higher than the frequency in NTM in our cohort and in viruses undergoing sexual transmission while the frequency of PNGS-N295 in children was significantly lower than the frequency in TM and acute horizontal infections. Collectively, our results provide evidence that the presence of the PNGS-N339 site and absence of the PNGS-N295 site in the gp120 envelope confers an advantage to HIV-1 when considering MTCT.
\end{abstract}

\section{Introduction}

$\mathbf{T}$

RANSMISSION OF HIV-1 FROM mother to child accounts for a significant proportion of transmissions in those countries where antiviral therapy is still limited. For example, in 2009 over 370,000 children less than 15 years of age were infected with human immunodeficiency virus, with the vast majority via mother-to-child transmission (MTCT) (www .unaidstoday.org). Known maternal risk factors associated with MTCT are high plasma viral loads, low CD4 T cell numbers coinciding with advanced maternal immune deficiency, and prolonged labor. In the absence of antiretroviral therapy approximately a third of mothers will transmit HIV-1 to their children during pregnancy, labor, or through breastfeeding, and at equal ratios. ${ }^{1}$
A number of studies focused on analyzing the molecular characteristics of viruses that get transmitted, either through MTCT or sexual intercourse, show that the majority of infections are characterized by the presence of a highly homogeneous viral population. ${ }^{2}$ These viruses are predicted to exclusively utilize the CCR5 coreceptor (termed R5 viruses) for infection even when the mothers are shown to carry CXCR4 using variants (termed R5/X4 or X4 viruses), ${ }^{3}$ indicative of a strong R5 selection pressure. This is somewhat surprising in children given the predominance of CXCR4 expressing naive $\mathrm{CD}^{+}$lymphocytes in the circulation, a consequence of limited antigen exposure. ${ }^{4,5}$

Virus selection during MTCT may be influenced by the binding affinity of the gp120 envelope glycoprotein to a number of cell receptors. A higher affinity for the CD4

\footnotetext{
${ }^{1}$ Laboratory of Experimental Virology, Department of Medical Microbiology, Centre for Infection and Immunity Amsterdam (CINIMA), Academic Medical Centre of the University of Amsterdam, Amsterdam, the Netherlands.

${ }^{2}$ IATEC, International Antiviral Therapy Evaluation Center, Amsterdam, the Netherlands.

${ }^{3}$ CHUK, Centre Hospitalier Universitaire de Kigali and Belgian Technical Cooperation, Kigali, Rwanda.

*Present affiliation: International Centre for Reproductive Health, Department of Obstetrics and Gynaecology, Ghent University, Ghent, Belgium.
} 
receptor or the CCR5 coreceptor may be of advantage for the infecting virus particle. A low net charge within the V3 variable loop of gp120 and the presence of a potential $\mathrm{N}$ linked glycosylation site (PNGS) on position 301 of the V3 loop ${ }^{6-8}$ are variables that have been associated with higher CCR5 affinity and being the variants preferentially transmitted. 9,10

In MTCT the HIV-1 variants transmitted to children tend to be more resistant to maternal plasma at time of delivery in comparison to viruses from the mother. ${ }^{11,12}$ Some studies suggest that the properties and function of maternal antibodies (Abs) in MTCT can prevent perinatal transmission or mediate viral selection. ${ }^{13}$ A number of gp120 envelope characteristics have been associated with resistance against neutralizing Abs, such as the V1-V2 region of the envelope, which has been postulated to regulate neutralization sensitivity by occluding conserved epitopes such as the CD4 and/or coreceptor binding sites. ${ }^{14,15}$ The V1-V2 length of subtype C viruses has been shown to positively correlate with resistance to broadly cross-neutralizing sera. ${ }^{16-21}$

It has been postulated that HIV-1 transmission can be enhanced by binding of the gp120 glycan moiety to the C-type lectin DC-SIGN, which is expressed at high levels on immature dendritic cells (DCs) and facilitates infection of $\mathrm{CD}^{+} \mathrm{T}$ lymphocytes either locally or in distal lymph nodes. ${ }^{22-24} \mathrm{DC}-$ SIGN expressed by a subset of B cells in the tonsils and blood may also contribute to the infection of $\mathrm{CD} 4{ }^{+}$lymphocytes. ${ }^{25}$ If this mode of transmission plays a role in MTCT, selection of the transmitting virus may be driven by more efficient binding of the virus to DC-SIGN.

Here we study a group of 30 pregnant mothers from Rwanda infected with either HIV-1 subtype A or C viruses of whom seven transmitted virus to their children. No correlation was identified between viral loads or CD4 ${ }^{+}$lymphocyte counts for transmitting mother (TM) or nontransmitting mother (NTM). We analyzed the gp120 envelope sequences for TM, NTM, and infected children. From this analysis we identified two potential glycosylation sites, PNGS-N234 and PNGS-N339, which were associated with viruses that are preferentially transmitted from mother to child and a disadvantage in transmission for viruses with PNGS-N295.

\section{Materials and Methods}

\section{Study population}

This is a subanalysis as part of a clinical study performed in Kigali, Rwanda as previously described. ${ }^{26}$ In brief, the study is composed of a group of $30 \mathrm{HIV}-1$-infected women from Rwanda and their children. Plasma samples were obtained from both mothers and children at time of delivery and at week 16. Seven children tested HIV-1 positive at 16 weeks after delivery. All the women were given a single dose of nevirapine at the start of labor to reduce peripartum transmission of HIV-1. All children were breastfed during the 16week period.

\section{Viral load and $C D 4^{+}$cell count assessment}

Blood plasma samples were analyzed for HIV-1 viral loads using the Roche diagnostics RNA PCR quantification assay version 1.5, with a lower limit of detection of 400 copies $/ \mathrm{ml}$. Immunologic testing of mothers was performed in two laboratories (National HIV / AIDS Reference Laboratory, Kigali,
Rwanda and the Joint Clinical Research Center JCRC, Kampala, Uganda).

\section{HIV-1 RNA extraction}

HIV-1 RNA was isolated from blood plasma samples according to the method of Boom et al. ${ }^{27}$

\section{Children's infection status at birth and week 16}

Plasma samples taken at birth were tested in a reverse transcriptase polymerase chain reaction (RT-PCR) amplification assay covering the HIV-1 gp120 V3 region. Initially, viral RNA was converted to cDNA using Superscript III Reverse Transcriptase (Invitrogen) and the 3' primer V3Not (GCG CGG CCG CCC CCT CTA CAA TTA AAA CTG TG), followed by PCR using Taq DNA Polymerase (Roche), 3' primer 3' V3Not and 5' primer 5' V3Not (GCG CGG CCG CAC AGT ACA ATG TAC ACA TGG). A nested PCR was subsequently performed using $5^{\prime}$ primer 5' KSI (ATA AGC TTG CAG TCT AGC AGA AGA AGA) and 3' primer 3' KSI (ATG AAT TCT GGG TCC CCT CCT GAG GA) and the PCR product was analyzed by agarose/ethidium bromide electrophoreses. At week 16 the children's HIV-1 infection status was determined using standard viral load assessment as described above.

\section{Amplification of the V1-V5 envelope region}

To amplify the V1-V5 envelope region viral RNA was converted into cDNA using the Superscript III Reverse Transcriptase Kit (Invitrogen) and 3' primer A1262 (CTG ACG GTA CAG GCC AGA CAA TTA TTG TC) followed by a PCR using the Expand Long Template PCR system (Roche), according to the manufacturer's instructions. For the first PCR 5' primer A1053 (GAA AGA GCA GAA GAC AGT GGC AAT GA) and 3' primer A1262 were used, and for the second PCR the 3' primer was A1322 (TCT TGG GAG CAG CAG GAA GCA C) and the 5' primer was A0358 (GAG GAT ATA ATC AGT TTA TGG GA).

\section{Sequencing and analysis}

PCR products were sequenced using the ABI PRISM Big Dye Terminator 1.1 Cycle Sequencing Kit and the ABI 3730 XL DNA Analyzer (Applied Biosystems). Sequences were assembled using the CodonCode Aligner program (CodonCode Corporation). Alignments were performed taking the translation codons into account and the sequences were translated into amino acids using the BioEdit program (Ibis Therapeutics). Analysis of the amplified region was performed utilizing the neighbor-joining (N-J) method of MEGA version 4. Positions containing an alignment gap were included for pairwise sequence analysis.

\section{Subtype determination}

To determine HIV-1 subtype the V1-V5 envelope PCR products were sequenced using primers 5' A0385 (GAG GAT ATA ATC AGT TTA TGG GA), 3’ A1322 (TCT TGG GAG CAG CAG GAA GCA C), 5’ A1360 (GAG CCA ATT CCY ATA CAT TAT TG), and 3'A1191 (ATG GGA GGG GCA TAC ATT GC). The Subtype Reference Alignments of the Los Alamos Database (www.hiv.lanl.gov/content/sequence/NEWALIGN/ align.htm) year 2005 were used as reference sequences. 


\section{Cloning and sequence analysis}

V1-V5 envelope PCR products were cloned into the PCRIITOPO vector (Invitrogen). For each subject and time point between 5 and 15 clones were sequenced using the primers $5^{\prime}$ SP6 (ATT TAG GTG ACA CTA TAG), 3’ T7 (TAA TAC GAC TCA CTA TAG G), 5' A1360, and 3' A1191. The number of PNGS was determined using the tool N-GlycoSite of the HIV sequence Database (http://www.hiv.lanl.gov/content/ sequence/GLYCOSITE/glycosite.html). The mean intrapatient diversity was calculated with the use of MEGA version 4 . The amino acid length and number of PNGS of the viral envelopes were compared using GraphPad Prism version 5.00 using the Mann-Whitney test, with $p$-values $<0.05$ being considered statistically significant. The overall V3 net positive charge was calculated by counting the number of positive charged amino acid residues $R$ and $K$ and the number of negative charged amino acid residues $\mathrm{D}$ and $\mathrm{E}$ being subtracted. ${ }^{28}$

\section{Direct sequencing of the C2-C3 region}

To expand the group of NTM for comparison of the frequency of PNGS-N295 and PNGS-N339 we performed direct sequencing on the V1-V5 envelope PCR products of 10 additional mothers encompassing amino acids 245 to 370 (HXB2 numbering) using 5' primer (GCG CGG CCG CAC AGT ACA ATG TAC ACA TGG) and 3' primer (AT TTC TAA GTC CCC TCC TGA).

\section{Comparison of the frequency of PNGS-N295 and PNGS-N339}

The percentages of glycosylation on pos. AA295 and AA339 (HXB2 numbering) for subtype A and C were calculated separately for the envelope sequences from the group of NTM, children infected in uterine (C2), ${ }^{29}$ children infected by breastfeeding (C3), ${ }^{30}$ breastfeeding TM (TM2), ${ }^{31}$ acute horizontal infections (contr.1), ${ }^{32-34}$ and a random selection of chronic infections acquired by horizontal transmission from the Search Interface of the HIV Sequence Database (contr.2) (http://www.hiv.lanl.gov/content/sequence/HIV/mainpage .html). In accord with the proportions of subtype A (5 out of 7 ) and C ( 2 out of 7 ) in our group of TM and children, percentages of individuals positive for glycosylation site N295 or N339 in the groups C2, C3, TM2, NTM, contr.1, and contr.2 were adjusted and the results analyzed using The Supervised Comparison of Subsets. ${ }^{35} p$-values $<0.05$ were considered statistically significant.

\section{Results}

High viral loads and low CD4 ${ }^{+} T$ cell counts were not the only determinants for HIV-1 transmission

Viral loads and $\mathrm{CD}^{+} \mathrm{T}$ cell counts were measured and compared for the 23 NTM versus seven TM (Table 1) (Fig. 1). Four mothers gave birth to HIV-1-positive children as determined by positive HIV-1 RT-PCR reactions. This indicates three of 26 mothers $(11.5 \%)$ were infected either intrapartum or during early breastfeeding, fitting with known HIV-1 transmission rates when mothers are given nevirapine at time of delivery. ${ }^{36}$ The TM were not found exclusively in the group with high viral loads and low $\mathrm{CD}^{+} \mathrm{T}$ cell counts. Three out of the seven TM had $\mathrm{CD}^{+}$cell counts $>500$ cells $/ \mu \mathrm{l}$, and three of seven had viral loads lower than 10,000 copies/ml, with one mother in both categories. These data suggest that factors other than viral load and $\mathrm{CD} 4^{+}$cell counts can influence HIV-1 transmission.

\section{Phylogenetic analyses of sequences of mothers and children}

The V1-V5 region of the gp120 envelopes of NTM and TM was sequenced. Out of the seven TM two $(29 \%)$ were infected with subtype C viruses while five (71\%) were infected with subtype A viruses. Of the group of 23 NTM, six (26\%) were infected with subtype $C$ and $15(65 \%)$ with subtype A viruses and for two mothers the virus subtype was undetermined.

We cloned and sequenced the V1-V5 regions of eight NTM and seven TM and their infected children. Phylogenetic analysis of the sequences of the V1-V5 regions of mothers and children confirmed the relatedness of the virus circulating in the respective mother-child pairs (data not shown). Four of the seven children were infected in utero of whom child 091

Table 1. Characteristics of Transmitting Mothers, NonTransmitting Mothers, and Children

\begin{tabular}{|c|c|c|c|c|c|c|c|}
\hline \multicolumn{5}{|c|}{ Mothers } & \multicolumn{3}{|c|}{ Children } \\
\hline Subject & $\begin{array}{l}\text { TM/ } \\
\text { NTM }\end{array}$ & Subtype & $\begin{array}{l}\text { Viral load day } 0 \\
\text { RNA cies } / m l\end{array}$ & $\begin{array}{l}\text { CD4 count day } \\
0 \text { cells } / \mu l\end{array}$ & Subject & PCR day 0 & $\begin{array}{l}\text { Viral load week } \\
16 \text { RNA cies } / \mathrm{ml}\end{array}$ \\
\hline 90 & $\mathrm{TM}$ & A & 203,333 & 250 & 91 & Pos. & 750,000 \\
\hline 100 & $\mathrm{TM}$ & A & 355,714 & 275 & 101 & Pos. & sna \\
\hline 130 & $\mathrm{TM}$ & C & 85,173 & 595 & 131 & Neg. & 7,590 \\
\hline 250 & $\mathrm{TM}$ & $\mathrm{C}$ & 3,214 & 206 & 251 & Pos. & 269,000 \\
\hline 290 & $\mathrm{TM}$ & $\mathrm{A}$ & 1,018 & 1168 & 291 & Pos. & 531,000 \\
\hline 300 & $\mathrm{TM}$ & A & 12,104 & 770 & 301 & Neg. & 747,000 \\
\hline 370 & $\mathrm{TM}$ & A & 8,803 & 289 & 371 & Neg. & 144,000 \\
\hline 50 & NTM & A & 12,703 & 258 & - & - & - \\
\hline 60 & NTM & A & 11,463 & 434 & - & - & - \\
\hline 110 & NTM & $\mathrm{A}$ & 8,329 & 990 & - & - & - \\
\hline 160 & NTM & A & 396,915 & 164 & - & - & - \\
\hline 190 & NTM & C & 1,900 & 342 & - & - & - \\
\hline 200 & NTM & $\mathrm{A}$ & 17,213 & 370 & - & - & - \\
\hline 340 & NTM & A & 6,827 & 546 & - & - & - \\
\hline 380 & NTM & A & 4,339 & 602 & - & - & - \\
\hline
\end{tabular}

TM, transmitting mothers; NTM, nontransmitting mothers who have been studied in detail. 


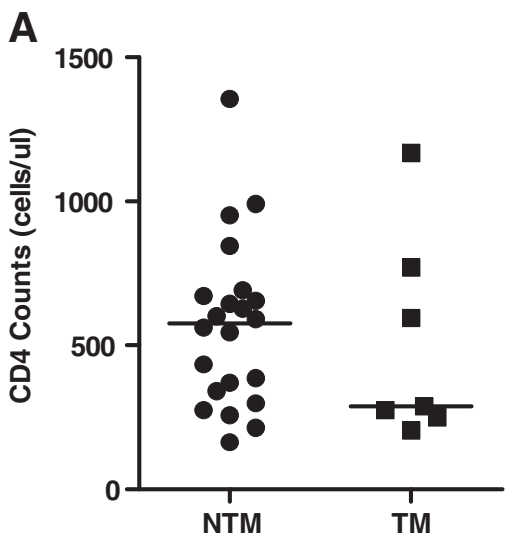

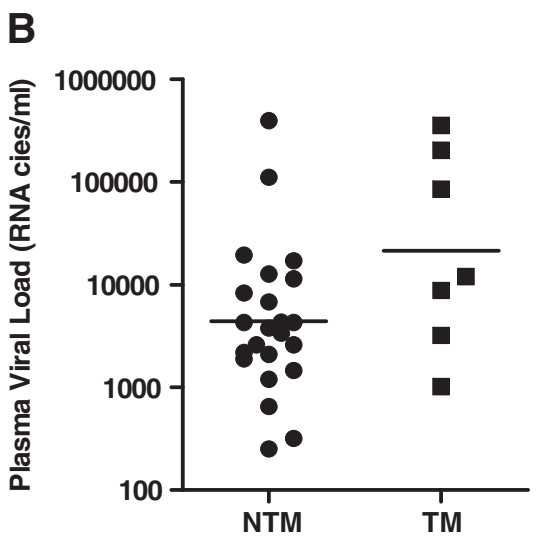

FIG. 1. Comparison of CD4 T cell counts and viral loads in transmitting mothers (TM) and nontransmitting mothers (NTM). The CD4 T cell counts in cells / $\mu \mathrm{l}$ (A) and the viral loads in number of copies/ml (B) from NTM and TM are not significantly different. $p$-values were calculated using the Mann-Whitney test. The horizontal lines indicate the median for each set. demonstrated two separate variants (Fig. 2A). All other infected children had only one HIV-1 variant while the viral quasispecies from the TM contained multiple variants (Fig. 2B). The intrapatient mean genetic distances of the V1-V5 region were analyzed (Fig. 3A). The mean genetic distance in
NTM was $0.022(95 \%$ CI $0.007-0.037 ; n=8)$ and was similar to that of TM $[0.025(95 \%$ CI $0.004-0.046 ; n=6)](p=0.82)$ or from the children [0.007 (95 CI 0.003-0.012; $n=6)](p=0.12)$; however, the mean intrapatient diversity in the children was significantly lower than in the TM $(p=0.007)$, which was in
A

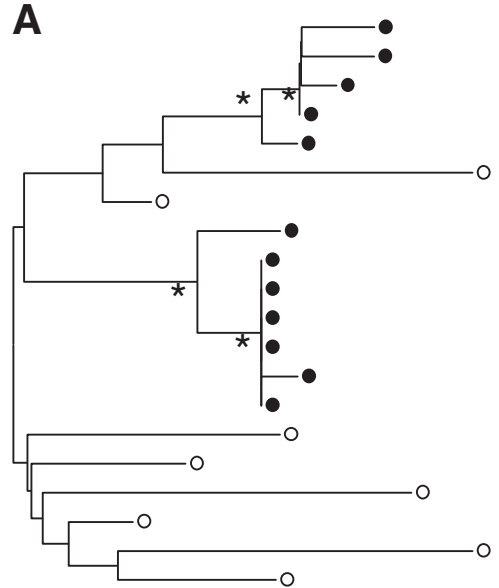

$\stackrel{\longmapsto}{\longrightarrow}$ Pair $90 / 91$

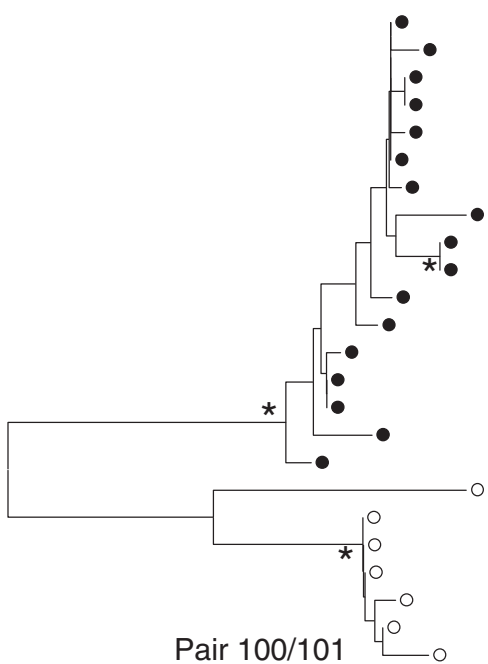

Pair 100/101

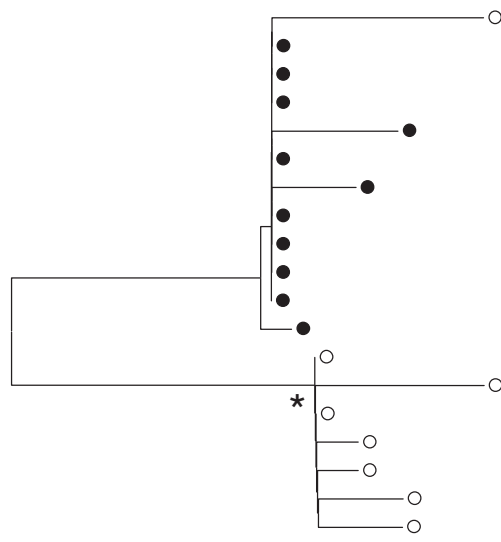

$\longdiv { 0 . 0 0 2 }$

Pair 250/251

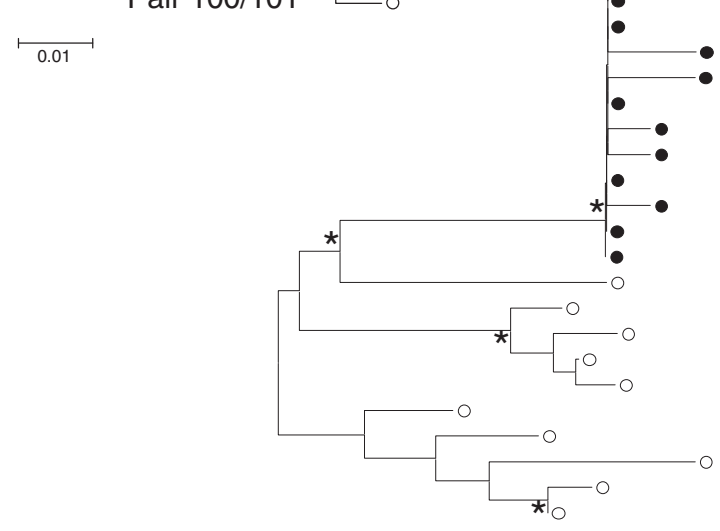

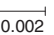

FIG. 2. Phylogenetic analysis of V1-V5 envelope sequences. The unrooted neighbor-joining trees show sequences of seven mother-child pairs. (A) Sequences of day of birth of four mother-child pairs of which the children tested positive at day of birth. (B) Sequences of 16 weeks after birth of three mother-child pairs of which the children tested negative at the day of birth, but positive at week 16. In one case transmission of two variants occurred (pair 090/091) and in the six other cases a single variant was transmitted. Mother's sequences are depicted with open circles and children's sequences with closed circles. Bootstrap values higher than 85 are indicated with an asterisk. 

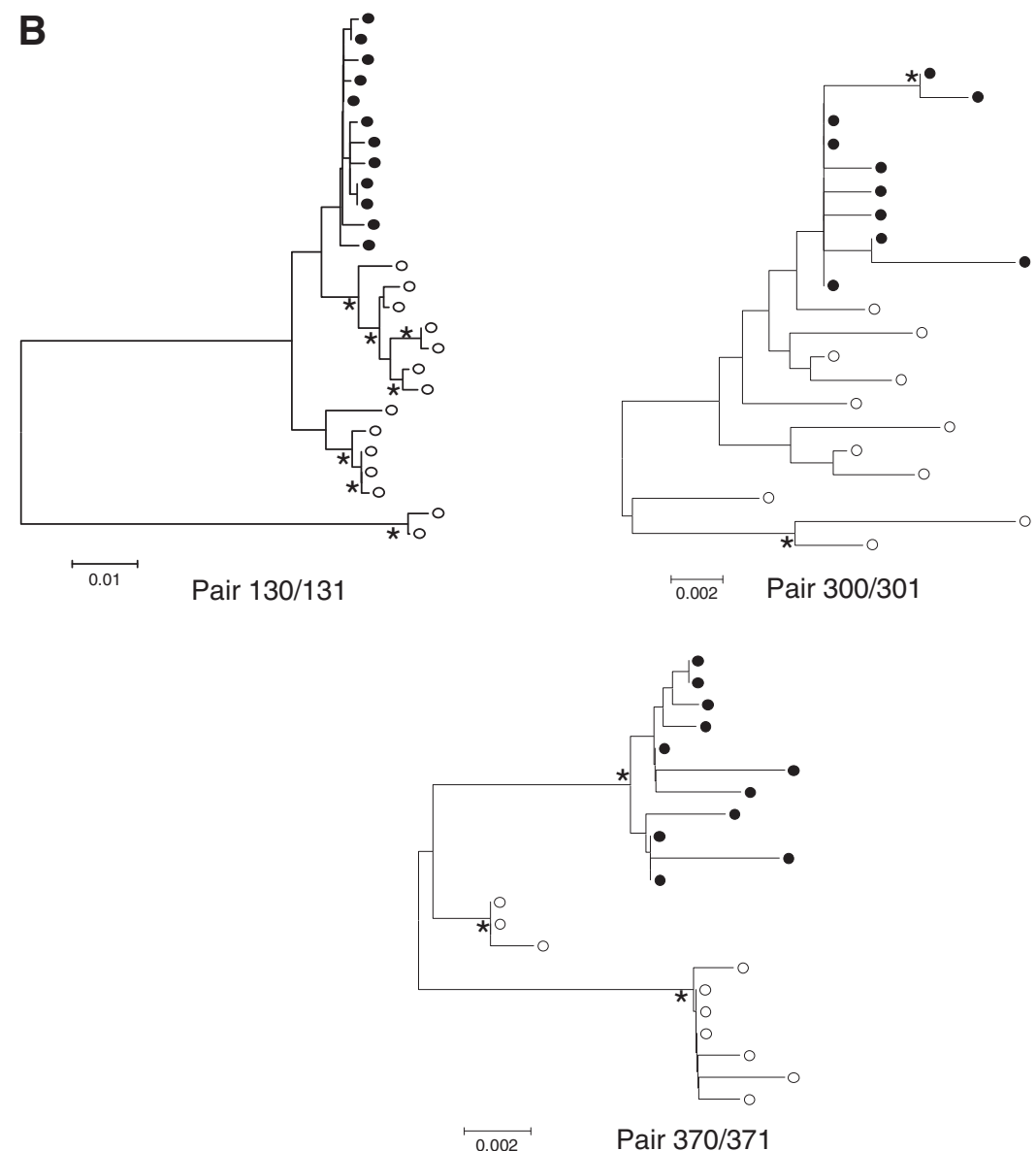

FIG. 2. (Continued).

agreement with the transmission of one virus variant in six out of seven children and two variants from one child.

V3 regions with a PNGS on position AA301 and a charge of +3 or +4 are transmitted

We next analyzed the number of PNGS and the overall V3 charge in the three groups. PNGS-N301 (HXB2 numbering), which is associated with CCR5 usage, was found in the majority of clones from TM $(100 \%$ in $6 / 7$ and $90 \%$ in $1 / 7)$ and NTM $(100 \%$ in $6 / 8,25 \%$ and $0 \%$ in $1 / 8)$, and was present in
$100 \%$ of the clones of all children. The mean V3 charges of viruses from NTM varied between 1.89 and 4.03 (average 3.17) and for TM between 2.58 and 5.00 (average 3.69) and was found to be narrower at between 3.00 and 3.91 (average 3.40) in the children (Fig. 3B).

\section{No differences in V1-V2 and V1-V5 length and number of PNGS}

To identify differences between the virus variants in the NTM and TM of children we analyzed the envelope
FIG. 3. Viral characteristics between NTM, TM, and children. (A) The mean intrapatient diversity of the V1-V5 envelope region in the children $(\mathrm{C})$ was significantly lower than in TM, but not significantly different from the NTM. $p$-values were calculated using the MannWhitney test. The horizontal lines represent the mean and the standard error of the mean. (B) The mean net charges of the V3 loop were not significantly different between NTM, TM, and children (C), but the range of the mean $\mathrm{V} 3$ charges of viruses from children was narrower than in the mothers. The horizontal lines represent the mean and the standard error of the mean. $p$-values were calculated using the Mann-Whitney test.

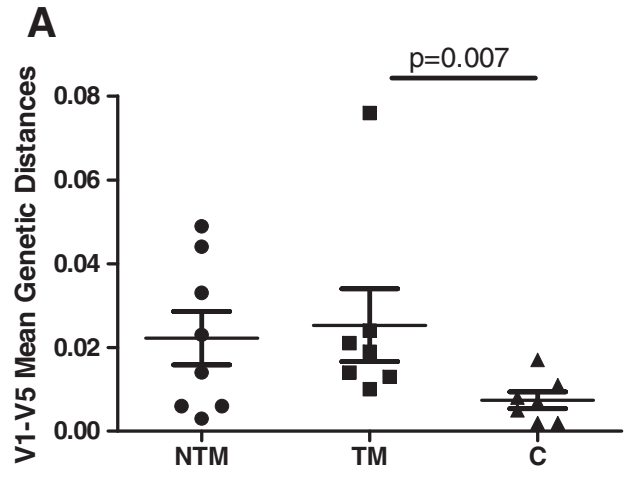

B

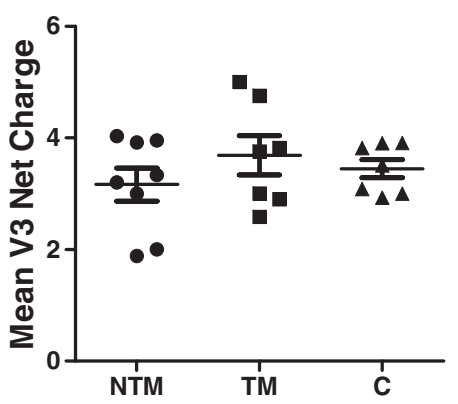



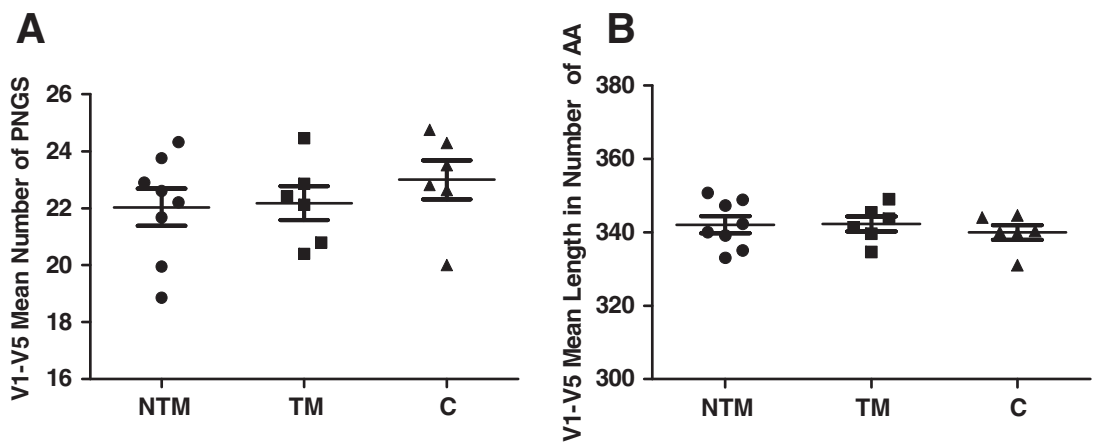

FIG. 4. V1-V5 envelope length and number of potential N-glycosylation sites (PNGS) in NTM, TM, and children. No significant differences in V1-V5 length (A) and number of PNGS (B) between NTM, TM, and children (C) are observed. $p$-values were calculated using the MannWhitney test. The horizontal lines represent the mean and the standard error of the mean.

sequences. We compared the size and the number of PNGS of the V1-V5 region and the V1-V2 region. The mean length of the V1-V5 region of amplified products from the NTM was 342.1 (95\% CI 336.6-347.5) amino acids, in the TM 342.3 (95\% CI 337.0-347.5) amino acids $(p=0.95)$, and in the children 340.0 (95\% CI 334.8-345.1) ( $p=0.70)$ amino acids (Fig. 4A). The mean number of PNGS in the V1V5 region of the viruses in the NTM was 22.0 (95\% CI 20.5-23.6), in the TM $22.2(95 \%$
CI 20.6-23.7) $(p=1.00)$, and in the children 23.0 (95\% CI 21.224.8) ( $p=0.39)$ (Fig. 4B). The mean size of the V1-V2 of NTM was 73.0 (95\% CI 66.4-79.7) amino acids, in the TM 68.8 (95\% CI 64.9-72.8) $(p=0.24)$ and in the children 68.6 (95\% CI 64.572.7) $(p=0.22)$. Overall, we did not find significant differences between the NTM, the TM, or the children in length and number of PNGS in either the V1-V2 or V1-V5 envelope regions.

Table 2. Variation in HIV-1 gr160 Potential N-Glycosylation Sites Between Nontransmitting Mothers, Transmitting Mothers, and Children

\begin{tabular}{|c|c|c|c|c|c|c|c|}
\hline \multicolumn{2}{|c|}{ Region HXB2pos. } & \multicolumn{2}{|c|}{ C2 PNGS-N234 } & \multicolumn{2}{|c|}{ C2-V3 PNGS-N295 } & \multicolumn{2}{|c|}{ C3 PNGS-N339 } \\
\hline Patient no. & Subtype & \# clones & $\%$ & \# clones & $\%$ & \# clones & $\%$ \\
\hline 91 & A & $12 / 12$ & 100 & $12 / 12$ & 100 & $12 / 12$ & 100 \\
\hline 101 & A & $15 / 15$ & 100 & $0 / 15$ & 0 & $15 / 15$ & 100 \\
\hline 131 & C & $12 / 12$ & 100 & $0 / 12$ & 0 & $12 / 12$ & 100 \\
\hline 251 & C & 11/11 & 100 & 11/11 & 100 & 11/11 & 100 \\
\hline 291 & A & $12 / 12$ & 100 & $12 / 12$ & 100 & $12 / 12$ & 100 \\
\hline 301 & A & $10 / 10$ & 100 & $10 / 10$ & 100 & $10 / 10$ & 100 \\
\hline 371 & A & $10 / 10$ & 100 & $0 / 10$ & 0 & $0 / 10$ & 0 \\
\hline
\end{tabular}

Transmitting Mothers

\begin{tabular}{lccccrr}
\hline Patient no. & Subtype & \# clones & $\%$ & \# clones & $\%$ & \# clones \\
\hline 90 & A & $8 / 8$ & 100 & $8 / 8$ & 100 & $8 / 8$ \\
100 & A & $6 / 6$ & 100 & $0 / 6$ & 0 & $6 / 6$ \\
130 & C & $14 / 14$ & 100 & $0 / 14$ & 0 & $9 / 14$ \\
250 & C & $8 / 8$ & 100 & $8 / 8$ & 100 & $1 / 8$ \\
290 & A & $6 / 10$ & 60 & $10 / 10$ & 100 & $8 / 10$ \\
300 & A & $11 / 11$ & 100 & $9 / 11$ & 82 & 13 \\
370 & A & $10 / 10$ & 100 & $0 / 10$ & 0 & $0 / 11$ \\
\hline
\end{tabular}

Nontransmitting Mothers

\begin{tabular}{lcccccc}
\hline Patient no. & Subtype & \# clones & $\%$ & \# clones & $\%$ & \# clones \\
\hline 50 & A & $6 / 6$ & 100 & $6 / 6$ & 100 & $0 / 6$ \\
60 & A & $16 / 16$ & 100 & $16 / 16$ & 100 & $7 / 16$ \\
110 & A & $9 / 9$ & 100 & $9 / 9$ & 100 & $8 / 9$ \\
160 & A & $12 / 12$ & 100 & $12 / 12$ & 100 & $3 / 12$ \\
190 & C & $0 / 11$ & 0 & $11 / 11$ & 100 & $11 / 11$ \\
200 & A & $0 / 8$ & 0 & $8 / 8$ & 100 & $0 / 8$ \\
340 & A & $0 / 19$ & 0 & $19 / 19$ & 100 & $0 / 19$ \\
380 & A & $12 / 12$ & 100 & $12 / 12$ & 100 & $0 / 12$ \\
\hline
\end{tabular}

\# clones, number of clones with a PNGS/number sequenced.

PNGS, potential N-glycosylation sites. 


\section{Selective transmission of viruses with a PNGS} on N234 and N339

To further identify envelope PNGS differences we mapped the sites in NTM, TM, and children. Six PNGS were found conserved in all variants for all groups (HXB2 numbering N136, N156, N198, N262, N332, and N447), with three sites showing a difference between the groups (Table 2). PNGS-N234 (in C2) was present in all of the children's clones, in the majority of the clones of TM (86\%), and less abundant in the NTM (62\%). Only $60 \%$ of the clones of TM 290 had a PNGS at position N234, whereas $100 \%$ of her child's (291) clones possessed this PNGS, suggesting an advantage in transmission. PNGS-N295 (between C2 and V3) was present in all NTM clones, the majority of clones in four/seven TM, and in four/seven children, which would suggest a disadvantage for transmission; however, when the PNGS is present transmission can occur. Six of seven children demonstrated PNGS-N339 in all virus variants, which was present in the majority of clones from TM (five/seven) and was found in only two/eight NTM. PNGS-N339 was absent in all the clones of mother 370 and the corresponding child 371 . Only $13 \%$ of the clones of mother 250 held PNGS-N339, whereas her child (251) had $100 \%$ of viruses carrying this PNGS. The above findings suggest that variants with PNGSN234 and/or PNGS-N339 were preferentially transmitted and that variants with PNGS-N295 had a disadvantage.

To verify our above findings we compared our results with data from different sources (Search Interface of the HIV Sequence Database) where we could compare sequences of subtype A or $\mathrm{C}$ viruses from children infected in utero (C2) $N=40)$, from children infected through breastfeeding (C3/ $N=40$ ), as well as from mothers who transmitted the virus by breastfeeding $(\mathrm{TM} 2 / N=83)$. Data from the virus populations of NTM were not available in the database. We performed a direct sequence analysis of 10 additional NTM from our cohort to increase the overall study group size. Viral sequences from acute infection (AI) patients of subtype $A$ and $C$ were included to verify if this PNGS is associated with recently sexual intercourse-transmitted viruses (control $1 / N=90$ ). We also included a random selection of sequences from drug-naive African individuals of subtype A and C (control $2 / N=318$ ) to verify the prevalence of the PNGS at position 295 and 339 in individuals other than mothers or children (Fig. 5A and B, respectively). If more than one sequence of an individual was available we calculated the consensus of these sequences. We matched the percentages of individuals positive for the PNGS to equivalent proportions of subtype $\mathrm{A}$ and $\mathrm{C}$ in the groups of TM and children that we investigated (C1 and TM1). To determine significance we performed a Supervised Comparison of the Subjects with the children groups $\mathrm{C} 1, \mathrm{C} 2$, and $\mathrm{C} 3$ as the reference group for the $\mathrm{G}$ standard (Fig. 5).

Due to the fact that sequences of groups C2, C3, and TM2 did not encompass PNGS-N234 we could not analyze this PNGS. When analyzing further PNGS-N295 we found it to be present in $50-70 \%$ children $(C 1, C 2, C 3)$, which was significantly reduced when compared to TM2 (70\%) and the acute horizontal transmissions (control 1) (69\%) (both $p=0.001$ ), which is in concordance with a disadvantage in MTCT for envelopes carrying PNGS-N295 (Fig. 5A). For PNGS-N339 we found this site to be present in over $79-86 \%$ of the consensus sequences for both TM and children, whereas for NTM and control 1 and control 2 the frequency was significantly reduced $(43 \%)(p \leq 0.001), 44 \%(p \leq 0.001)$ and $51 \%(p \leq 0.001)$, respectively, indicating a strong positive selection in MTCT for virus variants with a PNGS on position AA339 in the C3 region of the viral envelope (Fig. 5B).

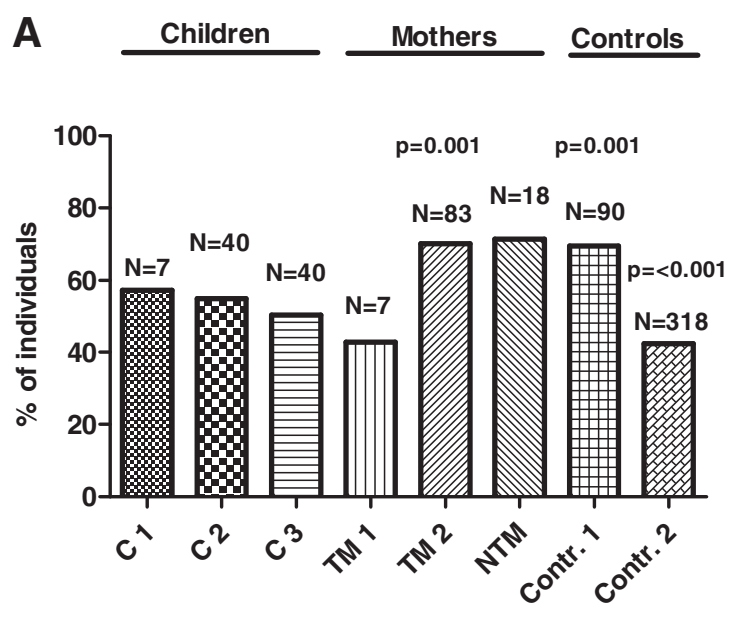

$\%$ of individuals positive for PNGS-N295

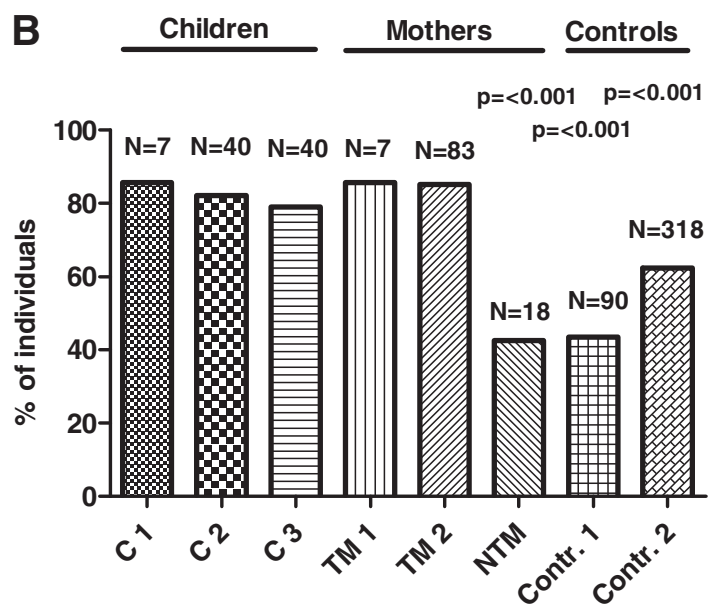

$\%$ of individuals positive for PNGS-N339

FIG. 5. PNGS percentages at positions (A) AA295 and (B) AA339 (HXB2 numbering). The subtype A and subtype C sequences from our TM and children were compared to an equivalent proportion of subtype A and subtype $C$ viruses from the literature. $\mathrm{C} 1$, children infected in uterine, perinatal, or by breastfeeding. C2, children infected in uterine. C3, children infected by breastfeeding. TM1, in uterine, perinatal, or by breastfeeding TM. TM2, by breastfeeding TM. NTM, nontransmitting mothers. Contr.1, acute horizontal infections. Contr.2, random selection of chronic infections acquired by horizontal transmission. The data for C1, TM1, and NTM are from our cohort; the data for C2, C3, TM2, Contr.1, and Contr.2 are from the Search Interface of the HIV Sequence Database. $p$-values were calculated using The Supervised Comparison of Subsets $^{35}$ with the children groups C1, C2, and C3 used as the reference group for the G standard. 


\section{Discussion}

From our V1-V5 gp120 sequence analysis of viruses preferentially transmitted between mothers and their children and in comparison to nontransmitting viruses we identified a higher frequency of PNGS at positions AA234 and AA339 and a lower frequency of PNGS at position AA295 in transmitted viruses. We confirmed the results of preferential transmission of viruses with PNGS at position N339 and the disadvantage in transmission for viruses with PNGS-N295 in MTCT through analyzing additional cohorts of MTCT as well as in individuals in their acute infection period and infected through sexual intercourse. We should emphasize that for PNGS-295 the observation is more group based than at the individual transmission pair level.

In sexual transmission HIV-1 subtype A and C variants with shorter envelope and fewer PNGS have been shown to be more efficiently transmitted and more susceptible to neutralizing antibodies present within the donor. ${ }^{37-39}$ One report concludes that variants transmitted from mother to child did not have shorter V1-V5 regions but did have fewer PNGS, ${ }^{11}$ while another study showed no association between transmitted variants and the length of the variable loops or the number of PNGS. ${ }^{40}$ We did not find differences in the number and the length of the V1-V2 and the V1-V5 regions between NTM, TM, and children in our cohort. Probably resistant viruses are preferentially transmitted from mother to child but it is not clear how changes in length of the envelope and in the number of PNGS correlate with MTCT. ${ }^{11,12,37,41,42}$

A low overall net charge and a PNGS at N301 within the gp120 V3 variable loop have been heavily associated with CCR5 usage. ${ }^{7}$ In agreement with other reports that show selection of CCR5 using variants in transmission we found that all transmitted viruses contained PNGS-N301 and that the V3 charge of these viruses was restricted to +3 and +4 , although the viruses of both mothers' groups had V3 charges ranging from +2 to +5 . The majority of studies to date have reported on viruses with low V3 charges being preferentially transmitted and here we describe a narrow range in V3 charges within children even when mothers contain quasispecies of viruses with lower charges. This could result from such viruses being the major variants in the TM, but could also reflect a less stable electrostatic interaction between gp 120 and the array of receptors to which it binds and which may influence HIV-1 transmission, including CD4, CCR5, or DCSIGN. ${ }^{39,43}$ PNGS N197, N230, N241, N301, N332, N386, N392, and N397 (HXB2 numbering) have been identified as candidates to play a role in DC-SIGN binding and may therefore exert such an influence through modulating this specific interaction. ${ }^{44,45}$

The selection for viruses with PNGS-N339 in viruses undergoing MTCT and not in acute horizontal infections could be explained by neutralizing antibody selection. Children will possess antibodies from the mothers whereas individuals sexually exposed to HIV-1 will not have such antibodies and can therefore be infected with viruses lacking PNGS-N339. The function of PNGS-N339 in the envelope of the children remains to be determined; however, PNGS-N339 is situated in the $\alpha_{2}$-helix region of $\mathrm{C} 3$ and several reports have demonstrated that residues within the $\alpha_{2}$-helix of subtype $C$ viruses are under strong positive selection and that unique mutational patterns in the $\alpha_{2}$-helix and acquisition of length in gp120 hy- pervariable domains are associated with resistance to autologous neutralization of HIV-1 subtype C. ${ }^{46}$ The disadvantage in MTCT for PNGS-N295 can be explained by the generation of resistance against neutralizing antibodies similar to $2 \mathrm{G} 12 .^{47}$

The further clarification of mechanisms of HIV-1 MTCT and the viruses involved will provide insights into which gp120 envelope regions may need to be targeted in a vaccine aimed at restricting transmission. Therapeutic vaccination of HIV-1-positive mothers during their pregnancy against such variants may have the benefit of restricting MTCT during all possible transmission periods and allow for lower rates of infection. We demonstrate that PNGS-N234 and PNGS-N339 within the gp120 envelope is associated with the risk of viral transmission from mother to child. Knowledge concerning which viruses are preferentially transmitted may aide in the future design of HIV-1 vaccine immunogens aimed at restricting MTCT.

\section{Acknowledgments}

The authors would like to thank the participants for their study involvement and Dr. J.M. Ruijter for assistance with the statistical analysis.

\section{Accession numbers}

GenBank accession numbers JN187678-JN187853 and JN097489-JN097554.

\section{Author Disclosure Statement}

No competing financial interests exist.

\section{References}

1. Kourtis AP, Lee FK, Abrams EJ, et al:: Mother-to-child transmission of HIV-1: Timing and implications for prevention. Lancet Infect Dis 2006;6(11):726-732.

2. Ometto L, Zanchetta M, Mainardi M, et al.: Co-receptor usage of HIV-1 primary isolates, viral burden, and CCR5 genotype in mother-to-child HIV-1 transmission. AIDS 2000; 14(12):1721-1729.

3. Clevestig P, Maljkovic I, Casper C, et al.: The X4 phenotype of HIV type 1 evolves from R5 in two children of mothers, carrying $\mathrm{X} 4$, and is not linked to transmission. AIDS Res Hum Retroviruses 2005;21(5):371-378.

4. Reinhardt PP, Reinhardt B, Lathey JL, and Spector SA: Human cord blood mononuclear cells are preferentially infected by non-syncytium-inducing, macrophage-tropic human immunodeficiency virus type 1 isolates. J Clin Microbiol 1995; 33(2):292-297.

5. Shalekoff S, Gray GE, and Tiemessen CT: Age-related changes in expression of CXCR4 and CCR5 on peripheral blood leukocytes from uninfected infants born to human immunodeficiency virus type 1-infected mothers. Clin Diagn Lab Immunol 2004;11(1):229-234.

6. de Jong JJ, de RA, Keulen W, Tersmette M, and Goudsmit J: Minimal requirements for the human immunodeficiency virus type $1 \mathrm{~V} 3$ domain to support the syncytium-inducing phenotype: Analysis by single amino acid substitution. J Virol 1992;66(11):6777-6780.

7. Hartley O, Klasse PJ, Sattentau QJ, and Moore JP: V3: HIV's switch-hitter. AIDS Res Hum Retroviruses 2005;21(2):171189. 
8. Pollakis G, Abebe A, Kliphuis A, et al.: Phenotypic and genotypic comparisons of CCR5- and CXCR4-tropic human immunodeficiency virus type 1 biological clones isolated from subtype C-infected individuals. J Virol 2004;78(6):28412852.

9. Matala E, Hahn T, Yedavalli VR, and Ahmad N: Biological characterization of HIV type 1 envelope V3 regions from mothers and infants associated with perinatal transmission. AIDS Res Hum Retroviruses 2001;17(18): 1725-1735.

10. Rainwater SM, Wu X, Nduati R, et al.: Cloning and characterization of functional subtype A HIV-1 envelope variants transmitted through breastfeeding. Curr HIV Res 2007;5(2): 189-197.

11. Wu X, Parast AB, Richardson BA, et al.: Neutralization escape variants of human immunodeficiency virus type 1 are transmitted from mother to infant. J Virol 2006;80(2):835844.

12. Blish CA, Blay WM, Haigwood NL, and Overbaugh J: Transmission of HIV-1 in the face of neutralizing antibodies. Curr HIV Res 2007;5(6):578-587.

13. Dickover R, Garratty E, Yusim K, et al.: Role of maternal autologous neutralizing antibody in selective perinatal transmission of human immunodeficiency virus type 1 escape variants. J Virol 2006;80(13):6525-6533.

14. Rong R, Bibollet-Ruche F, Mulenga J, et al.: Role of V1V2 and other human immunodeficiency virus type 1 envelope domains in resistance to autologous neutralization during clade C infection. J Virol 2007;81(3):1350-1359.

15. Pancera M, Majeed S, Ban YE, et al:: Structure of HIV-1 gp120 with gp41-interactive region reveals layered envelope architecture and basis of conformational mobility. Proc Natl Acad Sci USA 2010;107(3):1166-1171.

16. Chackerian B, Rudensey LM, and Overbaugh J: Specific Nlinked and O-linked glycosylation modifications in the envelope V1 domain of simian immunodeficiency virus variants that evolve in the host alter recognition by neutralizing antibodies. J Virol 1997;71(10):7719-7727.

17. Kwong PD, Wyatt R, Robinson J, et al.: Structure of an HIV gp120 envelope glycoprotein in complex with the CD4 receptor and a neutralizing human antibody. Nature 1998; 393(6686):648-659.

18. Pinter A, Honnen WJ, He Y, et al:: The V1/V2 domain of gp120 is a global regulator of the sensitivity of primary human immunodeficiency virus type 1 isolates to neutralization by antibodies commonly induced upon infection. J Virol 2004;78(10):5205-5215.

19. Sagar M, Wu X, Lee $S$, and Overbaugh J: Human immunodeficiency virus type $1 \mathrm{~V} 1-\mathrm{V} 2$ envelope loop sequences expand and add glycosylation sites over the course of infection, and these modifications affect antibody neutralization sensitivity. J Virol 2006;80(19):9586-9598.

20. Wyatt R, Moore J, Accola M, et al.: Involvement of the V1/ V2 variable loop structure in the exposure of human immunodeficiency virus type 1 gp120 epitopes induced by receptor binding. J Virol 1995;69(9):5723-5733.

21. Gray ES, Moore PL, Choge IA, et al.: Neutralizing antibody responses in acute human immunodeficiency virus type 1 subtype C infection. J Virol 2007;81(12):6187-6196.

22. Geijtenbeek TBH, Kwon DS, Torensma R, et al.: DC-SIGN, a dendritic cell specific HIV-1-binding protein that enhances trans-infection of T cells. Cell 2000;100(5):587-597.

23. Haase AT: Perils at mucosal front lines for HIV and SIV and their hosts. Nat Rev Immunol 2005;5(10):783-792.
24. Hladik F and McElrath MJ: Setting the stage: host invasion by HIV. Nat Rev Immunol 2008;8(6):447-457.

25. Rappocciolo G, Piazza P, Fuller CL, et al:: DC-SIGN on B lymphocytes is required for transmission of HIV-1 to T lymphocytes. PLoS Pathog 2006;2(7):e70.

26. Luchters SMF, Veldhuijzen NJ, Nsanzabera D, et al.: A phase I/II, randomized, placebo controlled study to evaluate chloroquine administration for reduction of HIV-1 RNA in breast milk in an HIV-infected breastfeeding population, The CHARGE study. XV International AIDS Conference, 11-16 July 2004, Bangkok, Thailand, PeB4499.

27. Boom R, Sol CJ, Salimans MM, et al.: Rapid and simple method for purification of nucleic acids. J Clin Microbiol 1990;28(3):495-503.

28. Pollakis G, Kang S, Kliphuis A, et al.: N-linked glycosylation of the HIV type-1 gp120 envelope glycoprotein as a major determinant of CCR5 and CXCR4 coreceptor utilization. J Biol Chem 2001;276(16):13433-13441.

29. Renjifo B, Chung M, Gilbert P, et al.: In-utero transmission of quasispecies among human immunodeficiency virus type 1 genotypes. Virology 2003;307(2):278-282.

30. Renjifo B, Fawzi W, Mwakagile D, et al.: Differences in perinatal transmission among human immunodeficiency virus type 1 genotypes. J Hum Virol 2001;4(1):16-25.

31. Koulinska IN, Villamor E, Msamanga G, et al.: Risk of HIV-1 transmission by breastfeeding among mothers infected with recombinant and non-recombinant HIV-1 genotypes. Virus Res 2006;120(1-2):191-198.

32. Salazar-Gonzalez JF, Bailes E, Pham KT, et al.: Deciphering human immunodeficiency virus type 1 transmission and early envelope diversification by single-genome amplification and sequencing. J Virol 2008;82(8):3952-3970.

33. Haaland RE, Hawkins PA, Salazar-Gonzalez J, et al.: Inflammatory genital infections mitigate a severe genetic bottleneck in heterosexual transmission of subtype A and C HIV-1. PLoS Pathog 2009;5(1):e1000274.

34. Abrahams MR, Anderson JA, Giorgi EE, et al.: Quantitating the multiplicity of infection with human immunodeficiency virus type 1 subtype $C$ reveals a non-poisson distribution of transmitted variants. J Virol 2009;83(8):3556-3567.

35. Schaaf GJ, Ruijter JM, van RF, et al.: Full transcriptome analysis of rhabdomyosarcoma, normal, and fetal skeletal muscle: Statistical comparison of multiple SAGE libraries. FASEB J 2005;19(3):404-406.

36. Ayouba A, Tene G, Cunin P, et al.: Low rate of mother-tochild transmission of HIV-1 after nevirapine intervention in a pilot public health program in Yaounde, Cameroon. J Acquir Immune Defic Syndr 2003;34(3):274-280.

37. Derdeyn CA, Decker JM, Bibollet-Ruche F, et al.: Envelopeconstrained neutralization-sensitive HIV-1 after heterosexual transmission. Science 2004;303(5666):2019-2022.

38. Chohan B, Lang D, Sagar M, et al.: Selection for human immunodeficiency virus type 1 envelope glycosylation variants with shorter V1-V2 loop sequences occurs during transmission of certain genetic subtypes and may impact viral RNA levels. J Virol 2005;79(10):6528-6531.

39. Keele BF and Derdeyn CA: Genetic and antigenic features of the transmitted virus. Curr Opinion HIV AIDS 2009;4(5):352-357.

40. Samleerat T, Braibant M, Jourdain G, et al:: Characteristics of HIV type 1 (HIV-1) glycoprotein 120 env sequences in mother-infant pairs infected with HIV-1 subtype CRF01_AE. J Infect Dis 2008;198(6):868-876.

41. Rong R, Li B, Lynch RM, et al.: Escape from autologous neutralizing antibodies in acute/early subtype C HIV-1 
infection requires multiple pathways. PLoS Pathog 2009;5(9):e1000594.

42. Moore PL, Ranchobe N, Lambson BE, et al:: Limited neutralizing antibody specificities drive neutralization escape in early HIV-1 subtype C infection. PLoS Pathog 2009;5(9): e1000598.

43. Margolis L and Shattock R: Selective transmission of CCR5utilizing HIV-1: The 'gatekeeper' problem resolved? Nat Rev Micro 2006;4(4):312-317.

44. Hong PWP, Nguyen S, Young S, et al.: Identification of the optimal DC-SIGN binding site on human immunodeficiency virus type 1 gp120. J Virol 2007;81(15):8325-8336.

45. Hong PW, Flummerfelt KB, de PA, et al.: Human immunodeficiency virus envelope (gp120) binding to DC-SIGN and primary dendritic cells is carbohydrate dependent but does not involve 2G12 or cyanovirin binding sites: Implications for structural analyses of gp120-DC-SIGN binding. J Virol 2002;76(24):12855-12865.

46. Rong R, Gnanakaran S, Decker JM, et al.: Unique mutational patterns in the envelope alpha 2 amphipathic helix and ac- quisition of length in gp120 hypervariable domains are associated with resistance to autologous neutralization of subtype $C$ human immunodeficiency virus type 1 . J Virol 2007;81(11):5658-5668.

47. Gray ES, Moore PL, Pantophlet RA, and Morris L: N-linked glycan modifications in gp120 of human immunodeficiency virus type 1 subtype $C$ render partial sensitivity to $2 \mathrm{G} 12$ antibody neutralization. J Virol 2007;81(19):10769-10776.

Address correspondence to: William A. Paxton K3-106

Laboratory of Experimental Virology Academic Medical Center University of Amsterdam Meibergdreef 15 Amsterdam, 1105AZ

The Netherlands

E-mail: w.a.paxton@amc.uva.nl 